\title{
Gel electrophoretic separation of proteins from cultured neuroendocrine tumor cell lines
}

\author{
NATALIA MIĘKUS ${ }^{1}$, ILONA OLĘDZKA ${ }^{1}$, ALINA PLENIS ${ }^{1}$, ZOFIA WOŹNIAK ${ }^{2}$, ANNA LEWCZUK ${ }^{2}$, \\ PATRYCJA KOSZAŁKA ${ }^{3}$, BARBARA SEROCZYŃSKA ${ }^{4}$ and TOMASZ BĄCZEK ${ }^{1}$
}

\author{
${ }^{1}$ Department of Pharmaceutical Chemistry, Medical University of Gdańsk, Gdańsk 80-416; ${ }^{2}$ Department of Endocrinology and \\ Internal Medicine, Medical University of Gdańsk; ${ }^{3}$ Department of Medical Biotechnology, Laboratory of Cell Biology; \\ ${ }^{4}$ Bank of Frozen Tissues and Genetic Specimens, Medical University of Gdańsk, Gdańsk 80-210, Poland
}

Received December 2, 2013; Accepted June 5, 2014

DOI: $10.3892 / \mathrm{mmr} .2014 .2864$

\begin{abstract}
Neuroendocrine tumors (NET) often develop asymptomatically and are detected at a late stage. Currently, there exist certain markers of NET that occur only in the advanced stages of the disease. Still, there is need to develop markers specific of the early stage of cancer development. Nevertheless, biomarkers are mostly low-abundant proteins and require separation from complex protein mixtures, which remains a major challenge. The goal of the present study was to optimize one-dimensional-polyacrylamide gel electrophoresis (1D-PAGE) for separation and comparison of protein composition from neuroendocrine tumor samples. 1D-PAGE was optimized by modification of the gel concentration and by comparison of different gel staining protocols. In addition, several steps prior to electrophoresis were carried out to purify and preliminarily reduce the complexity of the sample. The results of these optimization steps indicated that use of an albumin removal kit can considerably decrease the amount of albumin in the samples, thereby allowing to detect proteins of low abundance. Optimal separation of the sample was obtained using a $12 \%$ polyacrylamide gel. Furthermore, the use of silver staining allowed detection of proteins at nanogram levels, whereas for Coomassie Brilliant Blue staining, the detection limit was 10 times higher. Optimization of the sample preparation workflow and parameters of the electrophoretic separation allowed to reduce the complexity of the studied material and facilitated further identification of proteins of low abundance in the sample. This study demonstrated that analysis of the secreted proteome of NET cells by 1D-PAGE is a simple and suitable tool for the identification of potential NET protein biomarkers.
\end{abstract}

Correspondence to: Dr Tomasz Bączek, Department of Pharmaceutical Chemistry, Medical University of Gdańsk, Hallera 107, 80-416 Gdańsk, Poland

E-mail: tbaczek@gumed.edu.pl

Key words: electrophoresis, mass spectrometry, neuroendocrine tumors, cancer biomarkers, sample preparation

\section{Introduction}

At present, the identification of biological molecules, such as proteins or peptides, from complex biological matrices is of great interest for scientists. This is mainly related to the importance of these molecules in the diagnosis of numerous pathological states of the human organism. Through the comparison of protein composition between healthy and diseased organisms, we can gain useful knowledge for the development of therapeutic strategies. This approach has immense potential for the understanding of carcinogenic processes, since it allows identifying the specific proteins that are altered in tumor samples. These proteins are named biomarkers and are highly desirable nowadays, since the number of patients suffering from cancer is increasing $(1,2)$. The majority of research efforts has focused on rare, not well-characterized cancer types, such as neuroendocrine tumors (NET). The tumors of the neuroendocrine cell system are a group of neoplasms characterized by distinct phenotypes with respect to pathology, immunohistochemistry, and hormonal syndromes. They most commonly occur in the intestines, but are also found in the lungs and the rest of the body. Unfortunately, NETs are rarely diagnosed prior to metastasis and, therefore, the survival of diagnosed patients remains low. It is thus of great importance to develop tools that will help detecting NET at the early stage in the clinic (3-5).

The field of biology that aims to investigate the composition of all the proteins (proteome) in an organism, tissue or cell line, is called proteomics (6). Proteomics is a rapidly evolving field. It involves identification and quantitative analysis of the whole-protein composition of a certain sample, as well as investigations on the activity, function and localization of individual proteins (7). Proteomic approaches are increasingly used for the identification of specific protein signatures in different body fluids, mainly in order to gain new knowledge on protein profiles and potential ways to deal with life-threatening diseases such as cancer, heart or respiratory track diseases (8-11). The analysis of complex protein mixtures, such as samples collected from patients with tumors, either from the blood, serum, plasma, or supernatants from primary cell cultures, remains a great challenge for scientists. The first step in proteomic studies involves fractionation and reduction of the complexity of the protein sample, which renders it more 
amenable to extensive analysis (12). Following separation of a mixture of proteins, the resulting fractions consist of fewer proteins, or peptides are created. Hence, the preliminary steps in the preparation of a sample are crucial for further identification of potential biomarkers for early cancer detection (13).

One-dimensional (1D)-polyacrylamide gel electrophoresis (PAGE), also known as sodium dodecyl sulphate (SDS)-PAGE, is the core analytical separation technique in proteomic studies. Since Tiselius' pioneering study in 1937 (14), which introduced a moving boundary method as an analytical tool for the electrophoretic study of proteins, electrophoretic methods have considerably diversified. 2D-PAGE was introduced more than two decades ago, and became widely used in proteomic studies (15). Nevertheless, 1D-PAGE is still used to separate complex macromolecule samples. This is due to the fact that the facilities necessary for separation in 1D gel electrophoresis are not sophisticated and expensive in comparison to 2D-separating technique facilities. However, despite its numerous advantages, 1D gel electrophoresis is a tool that cannot be comprehensively used in proteomic studies, since it is time-consuming, labor-intensive and semi-quantitative. Nevertheless, for complex protein samples such as body fluid samples from patients suffering from cancer, 1D-PAGE can provide important basic information on the protein composition of a sample.

The main goal of the present study was to optimize 1D-PAGE for separation and comparison of protein composition between complex protein mixtures. We investigated the supernatants from primary cell cultures (an heterogeneous mix of cells derived directly from the living tissue) generated from endocrine tumors. In each of the investigated samples, tumor cells secrete proteins (e.g. insulin) and peptides to the cell culture medium. In addition, several proteins necessary for cell culturing are presented in the medium. Therefore, the assumption was made that the samples are highly complex, and proteins and peptides require separation prior to further investigation aiming to identify cancer biomarkers.

In the present study, optimization of 1D-PAGE was achieved through gel modification to obtain the most appropriate, for the main aim of this survey, concentration of polyacrylamide. Moreover, different substances for staining the gels were tested. Furthermore, several steps prior to electrophoresis were undertaken to purify the sample, reduce its complexity and discard the most abundant proteins from the culture medium.

Efficient reduction of the complexity of the studied material considerably facilitates further identification of peptides and proteins, allowing to detect specific protein signatures of a disease. Numerous cancer biomarkers and proteomic patterns useful for the choice of appropriate treatment strategies have been revealed via proteomic approaches (16-19). Still, a comprehensive proteomic study on NET proteins has not been undertaken to date. Thus, our results will contribute to a better understanding of NET and facilitate the early diagnosis of this rare type of cancer.

\section{Materials and methods}

Reagents. The protein molecular mass marker for SDS-PAGE Precision Plus Dual Color was obtained from Bio-Rad (Hercules, CA, USA), and the SDS molecular weight (MW) Size Standard for SDS-capillary gel electrophoresis (CGE) was obtained from Beckman Coulter Inc. (Fullerton, CA, USA). Biochemical-grade urea, SDS and $\beta$-mercaptoethanol were purchased from Bio-Rad. Highly purified water was obtained from the Milli-Q water purification system (EMD Millipore, Billerica, MA, USA). The Dodeca ${ }^{\mathrm{TM}}$ Silver Stain kit and Coomasie Briliant Blue G-250 for gel staining were purchased from Bio-Rad. The Calbiochem ${ }^{\circledR}$ ProteoExtract Albumin/immunoglobulin ( $\mathrm{IgG}$ ) removal kit was from Merck KGaA (Darmstadt, Germany).

Apparatus. Electrophoresis was carried out on Mini-PROTEAN ${ }^{\circledR} 3$ Cell apparatus (Bio-Rad). Liquid chromatography-tandem mass spectrometry (LC-MS/MS) analysis was performed using the nanoACQUITY Ultra Performance LC ${ }^{\circledR}$ system from Waters Corp. (Milford, MA, USA), coupled with an Orbitrap Velos Pro mass spectrometer (Thermo Fisher Scientific, Waltham, MA, USA).

NET samples. The supernatant samples were obtained from tumor primary cell cultures derived from 11 patients diagnosed with NET. Patients were selected for this study on the basis of medical history, clinical examination and laboratory investigation. All patients provided written informed consent. The Ethics Committee of the Medical University of Gdańsk (Gdańsk, Poland) approved the study protocol. Cultivation of primary tumor samples was conducted at the Department of Cell Biology, at the Medical University of Gdansk. The cultures were carried out in the incubator at $37^{\circ} \mathrm{C}$, with $5 \%$ $\mathrm{CO}_{2}$, under sterile conditions.

The stages of primary cell culture preparation were as follows: i) isolation of neuroendocrine tumors during surgery in sterile conditions. ii) Tumor samples were cultured in RPMI-1640 medium supplemented with L-glutamine (CytoGen Corp., Princeton, NJ, USA) and incubated for $20 \mathrm{~min}$ at room temperature with a high concentration of antibacterial agents (penicillin, 1,000 units; streptomycin, $1 \mathrm{mg} / \mathrm{ml}$ ) in fresh RPMI-1640 medium without fetal bovine serum (FBS). iii) Tumor samples were placed on Petri dishes, fat and connective tissue were removed as much as possible, and the sample was minced with a scalpel. Minced fragments were pressed through a nylon cell strainer $(100 \mu \mathrm{m}$ mesh; BD Biosciences, San José, CA, USA) and suspended in RPMI-1640 supplemented with L-glutamine. The cell suspension was centrifuged ( $5 \mathrm{~min}$ at $300 \mathrm{x} \mathrm{g}$, at room temperature), and the pellet was subjected to osmotic shock in order to achieve hemolysis of the erythrocytes. Cells were centrifuged once. iv) The pellet was resuspended in full medium, i.e., cell culture medium containing FBS or serum-free cell culture medium. The cell culture medium containing FBS was prepared by adding $10 \%$ FBS, 100 units of penicillin and $100 \mu \mathrm{g} / \mathrm{ml}$ of streptomycin to RPMI-1640 medium containing L-glutamine at the concentration of $0.3 \mathrm{~g} / \mathrm{l}$. Serum-free cell culture medium was prepared by supplementing the RPMI-1640 medium (with L-glutamine) with the ITS liquid medium supplement (final concentration: $10 \mu \mathrm{g} / \mathrm{ml}$ insulin, $5.5 \mu \mathrm{g} / \mathrm{ml}$ transferrin, $29 \mathrm{nM}$ sodium selenite, $1 \mathrm{mM}$ of sodium pyruvate, $10 \mathrm{ng} / \mathrm{ml}$ of human epidermal growth factor, $0.1 \mathrm{nM}$ triiodothyronine, $50 \mathrm{nM}$ hydrocortisone, 100 units of penicillin and $100 \mu \mathrm{g} / \mathrm{ml}$ of streptomycin). The cells were counted, distributed at a density of $8 \times 10^{5}$ cells per well on 24 -well plates (Sarstedt AG \& Co, 
Table I. The list of the samples investigated in this study, obtained from cell cultures of samples from 11 NET patients (supernatants) and of control samples, containing cell culture medium.

Type of tumor, location

NET samples ${ }^{\mathrm{a}}$

Control samples ${ }^{\mathrm{a}}$

Non-functioning, non-secreting lung carcinoid

Non-functioning, non-secreting lung carcinoid

1 - Functioning, secreting pancreas primary carcinoid

2 - Metastatic lymph nodes, functioning

and secreting pancreas carcinoid

Non-functioning, non-secreting lung carcinoid

Primary gastric carcinoid

Non-functioning, non-secreting atypical lung carcinoid

Non-functioning, non-secreting gastric carcinoid

Functioning, non-secreting lung carcinoma

Non-functioning, non-secreting lung carcinoid

Non-functioning, non-secreting pancreas carcinoid

Non-functioning, non-secreting pancreas carcinoid
NET 1 sup. FBS
NET 2 sup. FBS
NET 3.1 sup. FBS
NET 3.2 sup. FBS
NET 4 sup. serum-free
NET 5 sup. FBS
NET 5 sup. serum-free
NET 6a sup. FBS
NET 6b sup. serum-free
NET 7 sup. serum-free
NET 8 sup. serum -free
NET 9 sup. serum-free
NET 10 sup. serum-free
NET 11 sup. serum-free

\begin{abstract}
NET 1 med. FBS
NET 2 med. FBS

n.a.

n.a.

NET 4 med. serum-free

NET 5 med. FBS

NET 5 med. serum-free

NET 6 a med. FBS

NET $6 b$ med. serum-free

NET 7 med. serum-free

NET 8 med. serum-free

NET 9 med. serum-free

NET 10 med. serum-free

NET 11 med. serum-free
\end{abstract}

${ }^{a}$ Numbers in each NET and control sample denote the patient. NET, neuroendocrine tumor; sup., supernatant; med., medium; FBS, fetal bovine serum; n.a., sample not available.

Nümbrecht, Germany) in $1 \mathrm{ml}$ of medium, either cell culture medium containing FBS, or serum-free medium. The medium was also added to the wells not containing cells to obtain a control of protein background resulting from the medium supplements (FBS or others) and their degradation at $37^{\circ} \mathrm{C}$. v) After $72 \mathrm{~h}$ of incubation, supernatants from the cultures were collected, cleared from cell debris by centrifugation and distributed into cryovials. They were named, based on the used culture medium, as NET sup. FBS and NET sup. serum-free, respectively. Medium cultured in wells not containing cells was separately collected, also centrifuged, and distributed into cryovials. The corresponding samples were named as NET med. FBS and NET med. serum-free, respectively. Cryovials were preserved in liquid nitrogen. Table I presents the list of all samples analyzed in the study.

Sample preparation. Preparation of the collected biological material for further study involved the following procedures:

Albumin removal using the ProteoExtract Albumin/IgG removal kit. The ProteoExtract Albumin/IgG removal kit was used to remove albumin from the supernatants and the cell medium containing FBS. In both cases, the tested sample was thawed and mechanically shaken. Then, $450 \mu \mathrm{l}$ of binding buffer were added to $150 \mu \mathrm{l}$ of the tested sample and mixed. Next, the diluted sample was passed through a cartridge that was conditioned, according to the procedure provided in the kit, prior to sample loading. The resulting filtrate was collected in a separate tube. Moreover, the cartridge was washed with the binding buffer $(2 \times 200 \mu \mathrm{l})$, and these fractions were combined with earlier collected ones in sample collection tubes. Finally, the whole resulting filtrate $(1,000 \mu \mathrm{l})$ was used for further study.
Precipitation and concentration of protein samples. All samples, supernatants and controls from primary cell cultures with FBS and serum-free medium, were subjected to an acetone-methanol cleanup step, designed to desalt and concentrate the samples. Four volumes of cold acetone/ $1 \mathrm{mM}$ $\mathrm{HCl}-$ methanol 1:1 (v/v) were added to 1 volume of the serum-free supernatant sample and the serum-free cell culture medium sample $(150 \mu \mathrm{l})$, as well as to the supernatant and control samples from cell cultures with FBS obtained after removing the albumin fraction. Then, the samples were shaken and incubated for $1 \mathrm{~h}$ at $-20^{\circ} \mathrm{C}$. Following centrifugation for $15 \mathrm{~min}$ at $4^{\circ} \mathrm{C}(15,000 \mathrm{x} \mathrm{g})$, the protein supernatant was separated and evaporated in a water bath $\left(45^{\circ} \mathrm{C}\right)$ under reduced pressure. The protein precipitate was dissolved in $60 \mu \mathrm{l}$ of deionized water. The resulting solution of concentrated proteins was transferred to an Eppendorf tube and stored at $-20^{\circ} \mathrm{C}$.

ID SDS-PAGE. Before analysis, samples were thawed and mixed with a buffer consisting of Tris- $\mathrm{HCl}$ (pH 8.6), $2 \%$ SDS, $40 \%$ glycerol, $\beta$-mercaptoethanol and $0.02 \%$ bromophenol blue. The buffer was mixed with the same volume of the test protein sample, and the mixture was incubated in a water bath at $100^{\circ} \mathrm{C}$ for $3 \mathrm{~min}$, and then centrifuged at $14,000 \mathrm{x} \mathrm{g}$ for $10 \mathrm{~min}$. Addition of glycerol increased the density of the sample and facilitated application to the gel, while bromophenol blue stained the proteins and allowed observation of the protein separation on the gel. Next, $15 \mu \mathrm{l}$ of each protein sample were loaded to the gel, and electrophoresis was conducted at $200 \mathrm{~V}$ for $1 \mathrm{~h}$. In addition, a protein standard was added in a ready-to-use format, which eliminates the need to reduce, pre-mix, or add loading dyes to the standard 
solution. The standard was subjected to strict quality controls on appropriate gels to ensure consistent band migration and intensity. Subsequently, separation was carried out on 5, 12 or $15 \%$ polyacrylamide gels (1.5 $\mathrm{M}$ Tris- $\mathrm{HCl}, \mathrm{pH} 8.8)$ to optimize protein separation in the supernatants from the tumor cell line cultures. The electrode buffer ( $\mathrm{pH} 8.35$ ) contained $0.025 \mathrm{M}$ Tris, $0.192 \mathrm{M}$ glycine and $0.1 \%$ SDS.

The separated proteins were visualized by use of different staining agents. The most commonly used method to stain the bands on a gel is immersing the gel in a solution of Coomassie Brilliant Blue. The method is not labor-intensive, but can only detect $0.1 \mathrm{mg}$ of protein. Use of staining solutions based on silver salts allows researchers to obtain higher sensitivity and detect proteins at the nanogram level. Nevertheless, it requires additional dyeing steps and controls for the entire process in comparison to Coomassie Brilliant Blue staining. However, it is much more sensitive, since silver ions enable detection of down to $0.5 \mathrm{ng}$ of protein, via binding to carboxyl groups and thiol. In the present study, the gels were stained with $1 \%$ Coomasie Brilliant Blue G-250 for $1 \mathrm{~h}$ according to the manufacturer's instructions, and then destained with a solution containing $10 \%$ ethanol and $10 \%$ acetic acid. For comparison, a silver-staining procedure was followed, strictly according to the instructions supplied with the Dodeca Silver Stain kit. Next, the gels subjected to the following steps using reagents and instructions supplied with the kit: fixation for $30 \mathrm{~min}$, sensitization for $30 \mathrm{~min}$, washing 3 times for $5 \mathrm{~min}$, staining for $20 \mathrm{~min}$, rinsing for $1 \mathrm{~min}$, developing for 10-30 min, and washing, $10 \mathrm{~min}$ later, for $10 \mathrm{~min}$. Finally, the gels were scanned on a HP Scanjet G3010 Photo Scanner (Hewlett-Packard, Boise, ID, USA), and selected fractions of the proteins were digested for further LC-MS/MS analysis.

LC-MS/MS analysis. The LC-MS/MS analyses were performed in the Mass Spectrometry Laboratory, Institute of Biochemistry and Biophysics, at the Polish Academy of Sciences (Warsaw, Poland) using the nanoACQUITY Ultra Performance Liquid Chromatography (UPLC) system, coupled to an Orbitrap Velos Pro mass spectrometer. Prior to LC-MS analysis, gel bands were subjected to the standard procedure of trypsin digestion, in which the proteins were reduced for $30 \mathrm{~min}$ in $56^{\circ} \mathrm{C}$ with $100 \mathrm{mM}$ dithiothreitol (Carl Roth $\mathrm{GmbH}$, Karlsruhe, Germany), alkylated with $55 \mathrm{mM}$ iodoacetamide (Sigma-Aldrich, St. Louis, MO, USA) for $45 \mathrm{~min}$ in the dark at room temperature, and digested overnight using trypsin solution (Sequencing Grade Modified Trypsin; Promega Corporation, Madison, WI, USA) in $25 \mathrm{mM}$ ammonium bicarbonate $(25 \mathrm{ng} / \mu \mathrm{l})$. Peptides were eluted from the gel using an aqueous solution of $0.1 \%$ trifluoroacetic acid (Sigma-Aldrich) and $2 \%$ acetonitrile (ACN) (J.T. Baker, Phillipsburg, NJ, USA). The resulting mixture was injected into an RP-18 pre-column (Waters Corp.), using a water solution containing $0.1 \%$ formic acid (FA) (Fluka; Sigma-Aldrich) as the mobile phase, then separated for $45 \mathrm{~min}$ on a nano-HPLC RP-18 column (internal diameter $75 \mu \mathrm{M}$; Waters Corp.) using an ACN gradient (0-30\%) in the presence of $0.1 \% \mathrm{FA}$, at a flow rate of $250 \mathrm{nl} / \mathrm{min}$. The column outlet was coupled to the electrospray ion source of the spectrometer, dynamically switched between the measurement modes MS and MS/MS. Fragmentation was carried out by collision-induced dissociation, allowing sequencing of the five most intense signals from each MS scan. The minimal intensity of the signal for sequencing was set to 10,000 counts.

\section{Results and Discussion}

Proteomic studies involve a complex set of procedures, and may be carried out in different ways. Despite using a number of complementary techniques to examine the entire human proteome, only $\sim 20 \%$ of the human proteins were identified, due to limitations in the used methods $(20,21)$. However, it is believed that the sequence number obtained from these methods is sufficient to determine the presence of proteins. The task of characterizing the overall structure of the examined molecules is difficult, because of the numerous changes occurring in the protein structures (e.g., mutations or post-translational modifications). Therefore, a high number of complementary analytical methods are adopted in proteomic studies (20). This allows the close examination of the proteome profile of a given sample.

Proper preparation of the analyzed material prior to separaton is of great importance in proteomic studies. Appropriate sample preparation is equally important, as well as proper gel polymerization and the preparation of electrolytes. Each type of electrophoretic technique requires different conditions for sample preparation. In all cases, the separated sample needs not to contain excessive amounts of salt, which, in the ionized form, alters the migration and broadens the peaks in the proteinograms (22).

In this study, the effect of several steps of sample preparation, different percentages of polyacrylamide gels and different methods to stain the gels on the quality of the results were investigated. Maintenance of cells in a culture was achieved under strict conditions, with regards to the temperature, humidity, $\mathrm{pH}$ and composition of the medium. Cultures were conducted under sterile conditions, thus protecting the cells from microbial infection. For the primary cell culture, the addition of antifungal agents was avoided, since it can be problematic. When conducting tests, we ensured strict adherence to the protocols in order to obtain comparable culture conditions for all the samples.

The present study used tumor samples for which the type (whether it is a primary tumor or metastasis), location (organ)and stage of development (e.g., advanced) were carefully recorded. It is important to note that protein identification depends on a number of factors, for instance, whether a protein exists in different isoforms (post-translational modifications such as glycosylation), undergoes mutations, and is bound to the plasma albumin or present in a free form.

In this study, supernatant samples from primary cell cultures of tumors isolated from 11 patients with diagnosed NET and control samples containing cell culture medium alone (incubated under the same conditions) were obtained according to the protocol described in the Materials and Methods section 'NET samples'. Next, these samples were prepared for 1D-PAGE analysis as described in the section 'Sample preparation'.

Effects of albumin removal and deprotenization. Since the samples were grown on media containing FBS, they are expected to contain albumin and other macromolecular proteins. It should be also noted that both the samples 


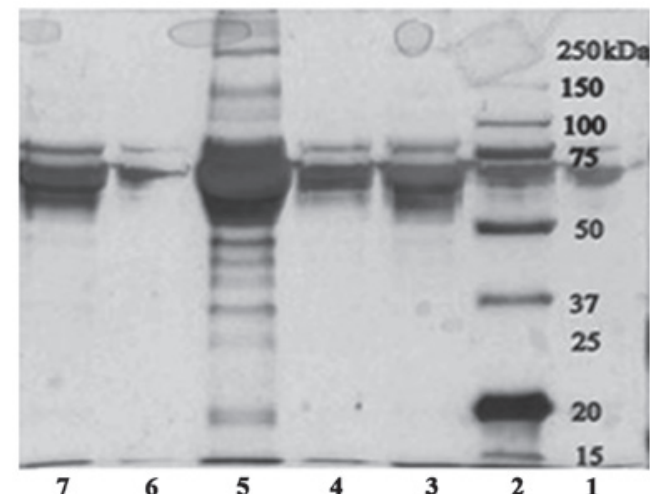

Figure 1. Typical proteinogram of the neuroendocrine tumor (NET) supernatant and control samples containing fetal bovine serum (FBS), separated by one-dimensional-polyacrylamide gel electrophoresis. The gel contain $12 \%$ polyacrylamide and was stained with silver salt. The samples were subjected to albumin removal and deprotenization prior to electrophoresis 1, Molecular weight (MW) standard of proteins in the 15-250 kDa range 2, sodium dodecyl sulphate MW standard; 3, NET 2 FBS medium; 4, NET 2 FBS supernatant; 5, NET 3.1 FBS supernatant; 6, NET 3.2 FBS supernatant; 7, NET 6a FBS medium.

containing FBS and the serum-free test samples were highly diluted, which can hinder detection of cancer biomarkers. For these reasons, we decided to remove from the serum samples the high-MW proteins, which may obscure the small-molecule protein fraction (23). This procedure was carried out only for supernatants and medium from primary cell cultures containing FBS. Removing albumin and immunoglobulin was achieved by using a specially designed set of spin columns filled with fluid that selectively retains macromolecular proteins. According to the manufacturer, the used ProteoExtract Albumin/IgG Removal kit can retain $>80 \%$ of albumin and immunoglobulins. Nevertheless, we need to consider that certain small proteins and peptides are bound to albumin and thus, will be depleted from the investigated sample with this procedure. Removal of serum albumin and $\mathrm{IgG}$ from samples is necessary, since it renders the samples less complex and enables detection of low-abundance proteins. In the next step, the samples containing FBS and serum-free samples were precipitated, and densification of the suspension was performed. This procedure was adopted in order to easily extract the proteins from the sample. Since the following steps need to be carried out on the soluble form of precipitated proteins, a cold precipitation reagent, composed of an acidified mixture of acetone $(1 \mathrm{mM} \mathrm{HCl})$ and methanol in a ratio of $1: 1$, was added to the protein sample. For deprotenization, the same sample volume of $150 \mu \mathrm{l}$ was used for both FBS and serum-free samples. However, the procedure of albumin and $\mathrm{IgG}$ removal required a final dilution of the FBS samples to $1,000 \mu \mathrm{l}$. Therefore, different volumes of the precipitate were used. Next, densification of the samples was performed by evaporation of the solvent on a thermostated water bath. Finally, the dry protein pellet was dissolved in $60 \mu \mathrm{l}$ of deionized water.

Figs. 1 and 2 present typical proteinograms of NET and control silver salt-stained samples obtained by 1D-PAGE. In the NET primary cell cultures containing FBS, distinct bands appeared in the MW range between 50 and $75 \mathrm{kDa}$ (Fig. 1). It is likely that these bands are derived from albumin, which

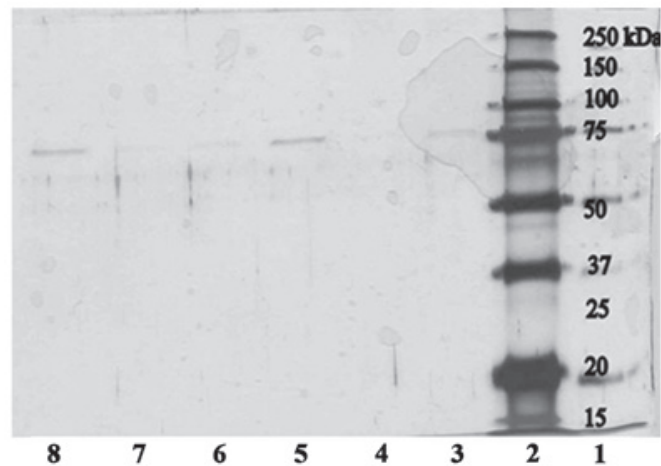

Figure 2. Typical proteinogram of the neuroendocrine tumor (NET) supernatant and control serum-free samples, separated by one-dimensional-polyacrylamide gel electrophoresis. The gel contains $12 \%$ polyacrylamide and was stained with silver salt. The samples were subjected to albumin removal and deprotenization prior to electrophoresis. 1, Molecular weight (MW) standard of proteins in the 15-250 kDa range; 2, sodium dodecyl sulphate MW standard; 3, NET 9 supernatant serum-free; 4, NET 7 supernatant serum-free; 5, NET 11 medium serum-free; 6, NET 8 medium serum-free; 7, NET 11 supernatant serum-free; 8 , NET 9 medium serum-free.

has a MW of $66.5 \mathrm{kDa}$. On the other hand, removal of serum albumin and $\mathrm{IgG}$ from the samples allowed observation of additional gel bands at small distance to the albumin band; these bands were not detected in the samples where the albumin fraction had not been removed (data not shown). Notably, in the sample NET 3.1 FBS supernatant (Fig. 1, lane 5), clear bands of high-concentration proteins and a wide range of MWs were observed. In the case of other samples from the patients with non-functioning NET and control samples grown on media containing FBS, such as NET 2 (Fig. 1, lanes 3 and 4) and NET 6a (Fig. 1, lane 7), only a few bands were observed in the range of 50-75 $\mathrm{kDa}$.

When the NET supernatants from serum-free primary cell cultures and control serum-free medium were analyzed by $1 \mathrm{D}-\mathrm{PAGE}$ (Fig. 2), faint protein bands in the range of 50-75 kDa were observed. Moreover, no significant differences were observed when comparing the NET 9 serum-free supernatant sample with the corresponding medium sample (lanes 3 and 8), as well as in the comparison of the NET 11 sample and control (lanes 7 and 5).

In conclusion, albumin removal and densification of the sample are two steps leading to a reduction in the sample volume and an increase in the protein concentration. These steps allow detecting the proteins that are present in a biological material in very low concentrations. These proteins can be further evaluated in terms of their value as NET biomarkers.

Effect of polyacrylamide percentage. 1D-PAGE is a widely used method for determination of the MW of proteins in denaturing conditions (24). The technique separates proteins due to their ability to move within an electrical current. By addition of the SDS detergent, the secondary and tertiary protein structures are lost. The SDS coats the proteins, mostly proportional to their $\mathrm{MW}$, and confers the same negative electrical charge across all proteins in the sample. Thus, the separation of proteins in a sample by 1D SDS-PAGE is only dependent on their MW.

The experimental protocol for 1D gel electrophoresis is relatively easy to follow and takes several hours. Choosing 


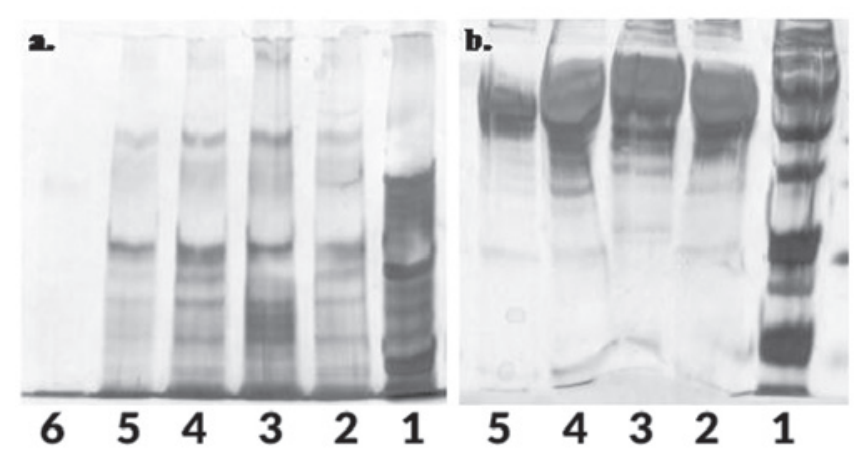

Figure 3. Typical proteinograms of the neuroendocrine tumor (NET) supernatant and control samples containing fetal bovine serum (FBS), separated by one-dimensional-polyacrylamide gel electrophoresis using (a) 5\% and (b) 15\% polyacrylamide. Proteins in both gels are stained with silver salt. (a) 1 , Sodium dodecyl sulphate molecular weight (MW) standard; 2, NET 3.1 supernatant FBS; 3, NET 5 supernatant FBS; 4, NET 6a supernatant FBS; 5, NET 4 supernatant serum-free; 6, NET 10 supernatant serum-free. (b) 1, SDS MW standard; 2, NET 3.1 supernatant FBS; 3, NET 5 supernatant FBS; 4 , NET 4 supernatant serum-free; 5, NET 10 supernatant serum-free.

the appropriate concentration of polyacrylamide can affect the polyacrylamide gel pore size. In addition, polyacrylamide gels are chemically inert; therefore they can be widely used as an analytical tool. Experiments testing different polyacrylamide concentrations in this study confirmed that the use of the appropriate polyacrylamide concentration is crucial for the success of the electrophoretic separation, since it affects the size of the separated protein molecules. The gels with dense cross-linking (percentage) of acrylamide/bis-acrylamide are suitable for the separation of proteins with low MWs. However, very high polyacrylamide concentrations may lead to exclusion of molecules with high MW from the gels. On the other hand, separation of proteins with high MWs can be achieved by using gels with low cross-linking of acrylamide/bis-acrylamide.

In this study, 5, 12 and $15 \%$ polyacrylamide gels were used. Figs. 1 and 2 illustrate the separation of proteins from NET samples using $12 \%$ polyacrylamide gels, while Fig. 3 shows typical proteinograms obtained on 5 and $15 \%$ acrylamide gels. As mentioned above, the 5\% gel (Fig. 3a) which has a lower concentration of polyacrylamide, allows effective separation of proteins of high MW (75-250 kDa). In the case of NET 3.1, 4, 5 and $6 \mathrm{a}$, proteins in the range $100-150 \mathrm{kDa}$ were observed. On the $15 \%$ gel (Fig. 3b) (containing a higher concentration of acrylamide, which entails a smaller pore size in the gel) the proteins of low MW (10-75 kDa) were accurately separated. Thus, for the same samples, accurate separation of proteins in the MW range $20-75 \mathrm{kDa}$ was obtained on the $15 \%$ gel. The serum-free sample (NET 10) was used here as a control, but no protein bands were detected on the $5 \%$ gel for this sample (Fig. 3a).

Taking these results into account, the best insight into the protein composition of the NET and control samples was obtained with $12 \%$ polyacrylamide gels, which offered the most selective separation of proteins within a specific MW distribution (Figs. 1 and 2). Thus, the concentration of $12 \%$ was selected as optimal for further 1D-PAGE analysis, since the present study was designed to separate as many proteins as possible in the complex biological samples.

In the next stage of the study, we tested the effect of different staining procedures on the visualization of proteins that may eventually be developed as biomarkers for the early diagnosis of NET.
Effect of staining procedure. Visualization of proteins in a gel is accomplished by staining techniques. Despite the availability of a wide variety of specific stains, the majority of 1D-PAGE gels is stained with Coomassie Brilliant Blue or some type of silver stain. Electrophoretic techniques in combination with further identification of proteins by mass spectrometry are very useful in proteomic studies. For proteomics work, protein stains need to be compatible with MS, and this requirement limits the choice of silver stains. The most interesting proteins in proteomic research are often low-abundance ones, but the concentrations of these proteins are near or below the detection limit of 1D-PAGE. Thus, the ideal staining method needs to allow maximum detection of minimum amounts of protein in a sample.

The protocol was further modified to test different staining methods. Although Coomassie Brilliant Blue G-250 is compatible with MS, the results revealed that the sensitivity of staining with this dye is lower than that obtained by staining with the Dodeca ${ }^{\mathrm{TM}}$ Silver stain (Fig. 4). This is because the concentration of the proteins in the investigated samples was low $(<0.1 \mu \mathrm{g} / \mathrm{ml})$. Proteins in such low concentration can not be visualized with the Coomassie Brilliant Blue dye. Silver staining is the most sensitive method for protein visualization, enabling detection of proteins in concentrations $<1 \mathrm{ng}$, whereas the detection limit for the Coomassie Brilliant Blue dye is within the microgram range. Nevertheless, considering that bands from the gels are commonly further analyzed by LC-MS, silver staining should be carefully performed, since it involves a multistep procedure, and the staining reaction has no endpoint. Moreover, proteins can be easily overstained, whereas the centers of the protein bands become lighter than the edges (plateau staining). In the present study, the Dodeca Silver ${ }^{\mathrm{TM}}$ Stain kit from Bio-Rad was used to ensure optimal conditions for staining of the proteins secreted by the NET cells that are present in very low concentration levels. According to the manufacturer, this kit is compatible with MS.

Finally, the protein bands obtained by 1D-PAGE of the supernatants of cultured samples from NET patients were analyzed by liquid chromatography coupled with mass spectrometry (LC-MS/MS) to identify the proteins expressed in NET tumors, which may represent potential biomarkers for the early diagnosis of this type of tumor. 


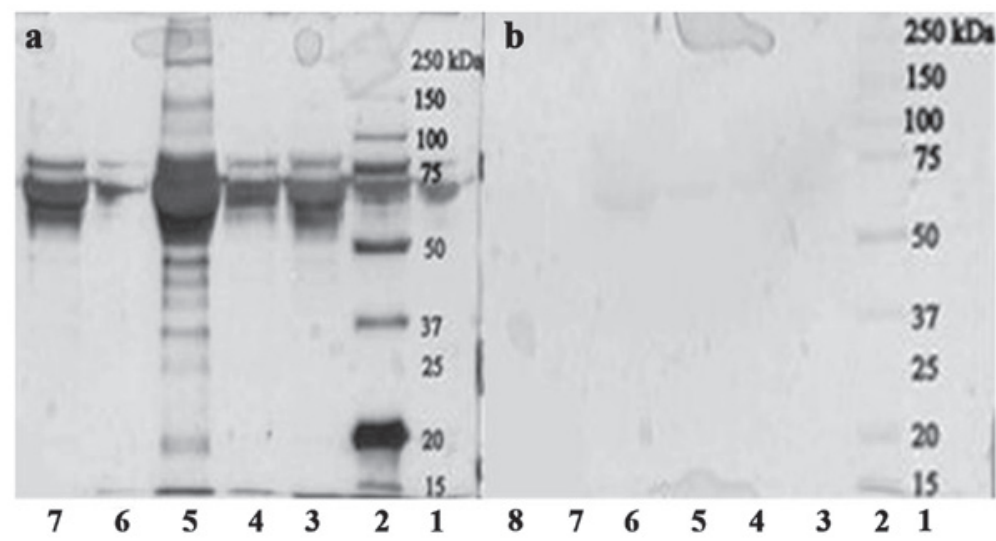

Figure 4. Typical proteinogram of the neuroendocrine tumor (NET) supernatant and control samples containing fetal bovine serum (FBS), separated by one-dimensional-polyacrylamide gel electrophoresis on a $12 \%$ polyacrylamide gel stained with (a) Dodeca ${ }^{\mathrm{TM}}$ Silver stain and (b) Coomassie Brilliant Blue G-250. (a) 1, Molecular weight (MW) standard of proteins in the 15-250 kDa range; 2, sodium dodecyl sulphate MW standard; 3 , NET 2 FBS medium; 4, NET 2 FBS supernatant; 5, NET 3.1 FBS supernatant; 6, NET 3.2 FBS supernatant; 7, NET 6 FBS medium. (b) 1, MW standard of proteins in the 15-250 kDa range; 2, SDS MW standard; 3, NET 2 FBS medium; 4, NET 2 FBS supernatant; 5, NET 3.1 FBS supernatant; 6, NET 3.2 FBS supernatant; 7, NET 6 supernatant serum-free; 8, NET 6 FBS medium.

LC-MS/MS analysis. 1D-PAGE does not allow identifying the proteins expressed in the tested sample. Thus, in order to detect NET biomarkers, the separated proteins were further analyzed by mass spectrometry.

As shown in Fig. 1, interesting 1D-PAGE profiles were obtained for the sample from a patient diagnosed with a hormone-secreting neuroendocrine tumor (NET 3.1, see also Table I), where additional protein bands were observed in relation to the samples coming from the non-secreting samples $(\mathrm{n}=3)$. These additional protein bands were further analyzed by LC-MS/MS. Raw data files were pre-processed with Mascot Distiller software (version 2.3; MatrixScience, London, UK). The obtained peptide masses and fragmentation spectra were matched to the National Center Biotechnology Information non-redundant database (17,351,384 sequences/5,948,435,699 residues) using the Mascot search engine (Mascot Daemon v. 2.3.0, Mascot Server v. 2.4.0, HYPERLINK 'http://www. matrixscience.com' MatrixScience). The following search parameters were applied: enzyme specificity was set to trypsin, peptide mass tolerance to $\pm 40 \mathrm{ppm}$ and fragment mass tolerance to $\pm 0.8 \mathrm{Da}$. The protein mass was left as unrestricted, and mass values as monoisotopic with one missed cleavage being allowed. Alkylation of cysteine by carbamidomethylation as fixed, and oxidation of methionine was set as a variable modification. Protein identification was performed using the Mascot search engine (MatrixScience), with the probability based algorithm. The expected value threshold of 0.05 was used for the analysis, which meant that all peptide identifications had $<1 / 20$ chance of being a random match. As expected, numerous proteins from the medium of the cell culture were identified in the supernatant of the tumour cell line culture of this sample, but LC-MS/MS analysis also allowed to identify a number of unique human proteins. The list of these proteins is presented in Fig. 5. Of particular importance may be the detection of two proteins (Fig. 6): the unnamed protein product, isolated from the NET 3.1 (5) band (GenBank GI number, 298880) and the histone macro H2A1.2, isolated from to the NET 3.1 (2) band (GI, 3493529). It is notable that this unique histone was also detected in a tumor metastasis sample from the same individual, NET 3.2 (data not shown). Histone macro H2A1.2 was previously detected in other tumor types, such as breast cancer (25). Its role in neuroendocrine malignances has not been investigated to date. Furthermore, chromogranin A, a known marker of NET (26), was also identified during direct LC-MS/MS analysis of the NET samples (data not shown).

In conclusion, 1D-PAGE conducted under denaturing conditions constitutes an important step in proteomic studies, prior to LC-MS analysis. It has the advantage of easy and rather fast separation of the proteins in complex protein mixtures. The electrophoretic process in our experiments did not alter the protein structure, and therefore, the presented data are reliable. In-gel digestion and LC-MS/MS analysis further allowed identification of peptides from specific proteins.

The data presented herein showed that optimization of the electrophoretic step is crucial for the final results, and can be performed either prior or during the process of electrophoresis. Before starting electrophoretic separation, one can conduct the cleaning and pre-fractionating steps, while during the separation, the constitution of the gel and the staining procedures can be modified to obtain optimal view of as many proteins in the complex mixture as possible.

There are still various aspects of proteomic protocols that need to be modified and optimized, and the separation step is one of these. Proteomic analysis approaches are especially useful in elucidating carcinogenic processes by identifying the specific proteins that are altered in tumor cells. Therefore, use of optimized proteomic protocols may allow isolating clinically important biomarkers for early cancer detection (27). This study demonstrated that analysis of the secreted proteome of neuroendocrine cell lines by 1D gel electrophoresis and LC-MS/MS is suitable for the identification of potential NET biomarkers. Initial assessment of NET samples identified certain unique proteins, the roles of which in NET merits further investigation.

\section{Acknowledgements}

The study was supported by the National Science Centre Project no. N N405 024340 and by the Ministry of Science and 


\begin{tabular}{|c|c|c|c|c|c|}
\hline & $\begin{array}{l}\text { Name } \\
\text { of } \\
\text { sample }\end{array}$ & MASCOT & Name of product & $\begin{array}{l}\text { Molecular } \\
\text { mass }\end{array}$ & ppm \\
\hline & $\begin{array}{l}\text { NET 3.1 } \\
\text { sup. FBS } \\
\text { (1) }\end{array}$ & - & - & - & - \\
\hline & & gi|3493529 & Histone macro H2A1.2 & 2126.0015 & 39 \\
\hline & $\begin{array}{c}\text { sup. FBS } \\
\text { (2) }\end{array}$ & gi $\mid 31645$ & $\begin{array}{c}\text { Glyceraldehyde-3-phosphate } \\
\text { dehydrogenase }\end{array}$ & $\begin{array}{l}1529.7872 \\
1762.7951\end{array}$ & $\begin{array}{l}27 \\
21\end{array}$ \\
\hline & & gi|4757756 & Annexin $\mathrm{A2}$ isoform 2 & 1243.6156 & 24 \\
\hline $250 \mathrm{kDa}$ & & gi|3294548 & Cathepsin $\mathrm{Z}$ precursor & 1009.4829 & 28 \\
\hline & NET 3.1 & gi|4502101 & Annexin A1 & $\begin{array}{l}1701.8785 \\
1549.8100\end{array}$ & $\begin{array}{l}20 \\
24\end{array}$ \\
\hline & $\begin{array}{c}\text { sup. FBS } \\
\text { (3) }\end{array}$ & gi|31645 & $\begin{array}{c}\text { Glyceraldehyde-3-phosphate } \\
\text { dehydrogenase }\end{array}$ & $\begin{array}{l}1410.7831 \\
1529.7872\end{array}$ & $\begin{array}{l}21 \\
24\end{array}$ \\
\hline & & gi|178855 & Apolipoprotein J precursor & 1872.9833 & 26 \\
\hline & NET 3, & gi|32015 & a-tubulin & 1714.9142 & 25 \\
\hline & $\begin{array}{c}\text { sup. FBS } \\
\text { (4) }\end{array}$ & gi|189520 & Pro- $\alpha-1$ type $V$ collagen & 2126.0936 & 37 \\
\hline$-\Rightarrow 15$ & & gi|180392 & $\alpha 1$ (I) chain propeptide & 1695.8250 & 36 \\
\hline & & gi|70058 & $\begin{array}{c}\text { Ig } \alpha-2 \text { chain } C \text { region - } \\
\text { human }\end{array}$ & 1212.6251 & 17.8 \\
\hline & NET 3.1 & gi|29888 & Unnamed protein product & 1420.7020 & 24.1 \\
\hline & (5) & gi|33700 & $\begin{array}{c}\text { Immunoglobulin } \lambda \\
\text { light chain }\end{array}$ & 989.5029 & 19 \\
\hline & & gi|29470 & $\begin{array}{c}\text { Human basement membrane } \\
\text { heparan sulfate } \\
\text { proteoglycan core protein }\end{array}$ & 1228.5619 & 20 \\
\hline
\end{tabular}

Figure 5. Liquid chromatography-tandem mass spectrometry results for the selected gel bands obtained by one-dimensional-polyacrylamide gel electrophoresis of the cell culture supernatant of a neuroendocrine tumor (NET) sample from patient 3. The second column presents the GenBank GI number of each protein, identified using the Mascot software. The last column shows the concentration of the protein in parts per million (ppm).

a gi|29888 unnamed protein product MW=1420.7020 peptide: K.WEIECPQYIR.K

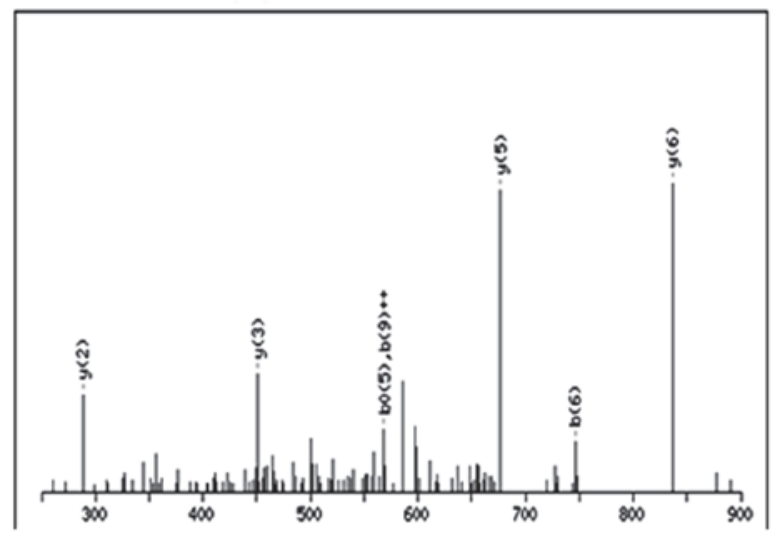

b gi|3493529histone macroH2A1.2 $M W=2126.0015$ peptide: K.AASADSTIEGTPADGFIVISTK.S

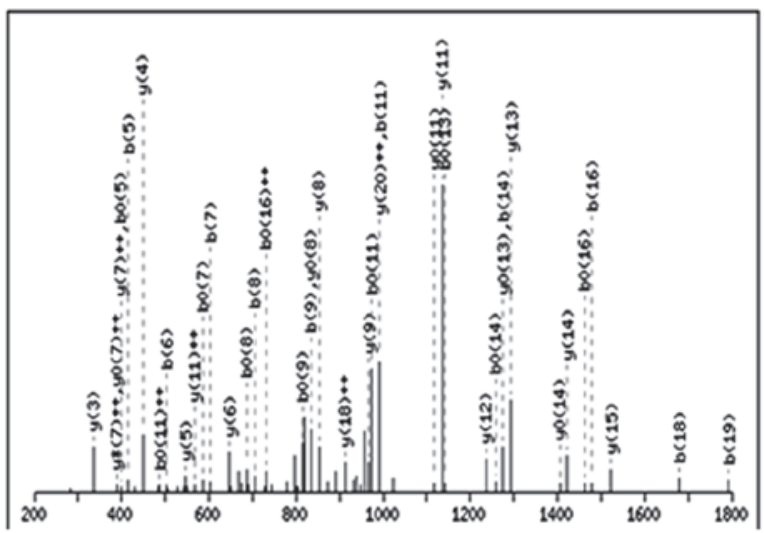

Figure 6. Example of fragment spectra of (a) unnamed protein product and (b) histone macroH2A1.2. The two peptides were identified by collision-induced dissociation in an OrbitrapVelos Pro apparatus. MW, molecular weight. 
Higher Education of the Republic of Poland, from the quality-promoting subsidy, under the Leading National Research Centre (KNOW) programme for the years 2012-2017.

\section{References}

1. Wulfkuhle JD, Liotta LA and Petricoin EF: Proteomic applications for the early detection of cancer. Nat Rev Cancer 3 267-275, 2003

2. Srinivas PR, Verma M, Zhao Y and Srivastava S: Proteomics for cancer biomarker discovery. Clin Chem 48: 1160-1169, 2002.

3. Klöppel G: Tumour biology and histopathology of neuroendocrine tumours. Best Pract Res Clin Endocrinol Metab 21: 15-31, 2007.

4. Jensen RT: Current diagnosis and management of gastrointestinal neuroendocrine tumours. Dig Liver Dis 33: 212-214, 2001.

5. Szczeblowska D: Diagnostics and treatment of neuroendocrine tumors of the digestive tract in the light of the present standards Pol Merkur Lekarski 22: 437-441, 2007 (In Polish).

6. Tyers M and Mann M: From genomics to proteomics. Nature 422 193-197, 2003

7. Anderson NL, Matheson AD and Steiner S: Proteomics: applications in basic and applied biology. Curr Opin Biotechnol 11: 408-412, 2003

8. Khalil AA: Biomarker discovery: a proteomic approach for brain cancer profiling. Cancer Sci 98: 201-213, 2007

9. Bergquist J, Baykut G, Bergquist M, Witt M, Mayer FJ and Baykut D: Human myocardial protein pattern reveals cardiac diseases. Int J Proteomics 2012: 342659, 2012.

10. O'Neil SE, Lundbäck B and Lötvall J: Proteomics in asthma and COPD phenotypes and endotypes for biomarker discovery and improved understanding of disease entities. J Proteomics 75: 192-201, 2011

11. de Noo ME, Tollenaar RA, Deelder AM and Bouwman LH: Current status and prospects of clinical proteomics studies on detection of colorectal cancer: hopes and fears. World J Gastroenterol 12: 6594-6601, 2006.

12. Issaq HJ, Conrads TP, Janini GM and Veenstra TD: Methods for fractionation, separation and profiling of proteins and peptides. Electrophoresis 23: 3048-3061, 2002.

13. Matt P, Fu Z, Fu Q and Van Eyk JE: Biomarker discovery: proteome fractionation and separation in biological samples. Physiol Genomics 33: 12-17, 2008

14. Tiselius A: A new apparatus for electrophoretic analysis of colloidal mixtures. Trans Faraday Soc 33: 524-531, 1937.
15. Wang $\mathrm{H}$ and Hanash S: Multi-dimensional liquid phase based separations in proteomics. J Chromatogr B Analyt Technol Biomed Life Sci 787: 11-18, 2003.

16. Ralhan R, Desouza LV, Matta A, Chandra Tripathi S, Ghanny S, Datta Gupta S, et al: Discovery and verification of head-and-neck cancer biomarkers by differential protein expression analysis using iTRAQ labeling, multidimensional liquid chromatography, and tandem mass spectrometry. Mol Cell Proteomics 7: 1162-1173, 2008.

17. Lawrie LC, Fothergill JE and Murray GI: Spot the differences: proteomics in cancer research. Lancet Oncol 2: 270-277, 2001.

18. Petricoin EF, Ardekani AM, Hitt BA, Levine PJ, Fusaro VA, Steinberg SM, et al: Use of proteomic patterns in serum to identify ovarian cancer. Lancet 359: 572-577, 2002.

19. Schiess R, Wollscheid B and Aebersold R: Targeted proteomic strategy for clinical biomarker discovery. Mol Oncol 3: 33-44, 2009.

20. Anderson NL, Polanski M, Pieper R, Gatlin T, Tirumalai RS, Conrads TP, et al: The human plasma proteome: a nonredundant list developed by combination of four separate sources. Mol Cell Proteomics 3: 311-326, 2004

21. Omenn GS, States DJ, Adamski M, Blackwell TW, Menon R, Hermjakob $\mathrm{H}$, et al: Overview of the HUPO Plasma Proteome Project: results from the pilot phase with 35 collaborating laboratories and multiple analytical groups, generating a core dataset of 3020 proteins and a publicly-available database. Proteomics 5: 3226-3245, 2005

22. Carpentier SC, Witters E, Laukens K, Deckers P, Swennen R and Panis B: Preparation of protein extracts from recalcitrant plant tissues: an evaluation of different methods for two-dimensional gel electrophoresis analysis. Proteomics 5: 2497-2507, 2005.

23. Millioni R, Puricelli L, Iori E and Tessari P: Rapid, simple and effective technical procedure for the regeneration of IgG and HSA affinity columns for proteomic analysis. Amino Acids 34: 507-509, 2008

24. Weber K and Osborn M: The reliability of molecular weight determinations by dodecyl sulfate-polyacrylamide gel electrophoresis. J Biol Chem 244: 4406-4412, 1969.

25. Li X, Kuang J, Shen Y, Majer MM, Nelson CC, Parsawar K, et al: The atypical histone macroH2A1.2 interacts with HER-2 protein in cancer cells. J Biol Chem 287: 23171-23183, 2012.

26. Nobels FR1, Kwekkeboom DJ, Coopmans W, Schoenmakers CH, Lindemans J, De Herder WW, et al: Chromogranin A as serum marker for neuroendocrine neoplasia: comparison with neuron-specific enolase and the alpha-subunit of glycoprotein hormones. J Clin Endocrinol Metab 82: 2622-2628, 1997.

27. Sardana G, Marshall J and Diamandis EP: Discovery of candidate tumor markers for prostate cancer via proteomic analysis of cell culture-conditioned medium. Clin Chem 53: 429-437, 2007. 